\title{
Aleitamento materno: estudo comparativo sobre o conhecimento e o manejo dos profissionais da Estratégia Saúde da Família e do Modelo Tradicional
}

\section{Maternal breastfeeding: a comparative study of knowledge and management among Family Health Strategy professionals and those using the traditional model}

Jamila Vasquez 1 Samuel C. Dumith 2

Lulie Rosane Odeh Susin 3

\begin{abstract}
Objectives: to evaluate and compare knowledge and quality of management of maternal breastfeeding $(M B)$ among professionals operating within the Family Health Strategy (FHS) and those working in basic health units using the traditional model, in the municipality of Rio Grande in the Brazilian state of Rio Grande do Sul, in 2012.

Methods: 269 professionals answered a questionnaire designed to evaluate their knowledge and management of $M B$ by way of scores of satisfactory, regular and unsatisfactory. Comparison was carried out using Fisher's exact test and Student's t test to compare the means. The analysis also examined the effect of various other variables independent of the model of care.

Results: only 22 (8.2\%) and 50 (18.6\%) of professionals attained a score of satisfactory for knowledge and management respectively, with FHS professional performing significantly better than those using the traditional model, $(p<0.05)$, with the exception of auxiliary nurses and technical assistants in the case of the management ( $p=0.05$ ).

Conclusions: the performance of professionals was found to fall short of expectations among those who deal directly with breastfeeding, especially those using the traditional model. This demonstrates the need for professional training as a way of effectively promoting the success of $M B$.
\end{abstract}

Key words Breast feeding, Health evaluation, Primary health care, Health personnel

\begin{abstract}
1 Programa de Pós graduação em Ciências da Saúde. Área Acadêmica da Saúde. Faculdade de Medicina. Universidade Federal do Rio Grande. Rua General Osório s.n. sala 425. Centro. Rio Grande, RS, Brasil. CEP 96200-400. E-mail: jamilavasquez@hotmail.com 2,3 Programa de Pós graduação em Ciências da Saúde. Faculdade de Medicina. Universidade Federal do Rio Grande. Rio Grande, RS, Brasil.
\end{abstract}

\section{Resumo}

Objetivos: avaliar e comparar o conhecimento $e$ a qualidade do manejo do aleitamento materno (AM) entre profissionais atuantes na Estratégia Saúde da Família (ESF) e nas unidades básicas de saúde com modelo tradicional, no Município de Rio Grande/RS, em 2012.

Métodos: foi aplicado aos 269 profissionais um questionário para avaliar o conhecimento e o manejo do AM por meio de escores classificados como satisfatório, regular e insatisfatório. A comparação das proporções se realizou através do Teste Exato de Fisher e o Teste t de Student foi utilizado visando à comparação das médias. Desenvolveu-se também análise de mediação controlando o efeito do modelo de atenção para algumas variáveis.

Resultados: somente 22 (8,2\%) e 50 (18,6\%) dos profissionais apresentaram desempenho satisfatório nos escores de conhecimento e manejo, respectivamente, tendo os trabalhadores da ESF melhores desempenhos, quando comparados aos profissionais do modelo tradicional, sendo essa diferença significativa $(p<0,05)$, exceto para os auxiliares e técnicos de enfermagem no escore de manejo $(p=0,05)$.

Conclusões: o desempenho dos profissionais mostrou-se aquém do esperado para quem lida diretamente com a amamentação, fato mais evidente no modelo tradicional. Dessa forma, comprova-se a necessidade de capacitação dos profissionais objetivando-se promover efetivamente o sucesso do AM.

Palavras-chave Aleitamento materno, Avaliação em saúde, Atenção primária à saúde, Profissional de saúde 


\section{Introdução}

As informações sobre os benefícios do aleitamento materno (AM), tanto para a saúde da mãe quanto para o desenvolvimento da criança, já estão amplamente difundidas. Segundo os conhecimentos científicos e as recomendações propostas pelos comitês internacionais de especialistas, a prática do aleitamento materno exclusivo (AME) nos primeiros meses de vida proporcionaria uma redução considerável nas taxas de mortalidade infantil.1,2 Apesar de as pesquisas nacionais sobre demografia e saúde apontarem o crescimento de tal prática no Brasil, essa tendência ainda está aquém do esperado. 3,4

No Brasil, a literatura mostra que alguns fatores podem influenciar negativamente o sucesso da amamentação exclusiva, favorecendo o desmame precoce. Entre eles destacam-se: uso de chupeta pelos lactentes, presença de fissura mamilar nas lactantes, 5 práticas hospitalares inadequadas, mudança da estrutura social acarretando o trabalho materno fora do lar, 6 ausência da mulher ao prénatal,5 depressão pós-parto, 7,8 além do nível de escolaridade da mãe, o qual influi na obtenção de esclarecimentos sobre a amamentação. ${ }^{7}$

Em contrapartida, o manejo clínico adequado da amamentação por parte dos profissionais da saúde é um fator importante e decisivo que aumenta a prevalência do AME. 9 Ele inclui não apenas conhecimentos básicos e habilidades em AM, mas também a competência do profissional na comunicação adequada por meio do aconselhamento, o qual tem o objetivo de ajudar as nutrizes a tomar decisões, após ouvi-las, entendê-las e dialogar sobre os prós e os contras das opções. 10

A rede básica de saúde (RBS) é a principal responsável pela assistência antes e depois do parto. As informações e o apoio oferecidos às gestantes no decorrer do pré-natal e na saída da maternidade dependem da atuação da RBS, sendo ela indispensável na promoção, proteção e apoio ao AME até os seis meses, prolongando-se aos dois anos ou mais. ${ }^{10}$ Assim, temos como estratégias à tríade mencionada a Norma Brasileira de Comercialização de Alimentos para Lactentes e Crianças de Primeira Infância, Bicos, Chupetas e Mamadeiras (NBCAL), a licença-paternidade, a obrigatoriedade do alojamento conjunto, o método mãe-canguru, os bancos de leite humano, a ampliação da licença-maternidade, além das Iniciativas Hospital Amigo da Criança e Unidade Básica Amiga da Amamentação. 10

Nos últimos anos, o Brasil vem investindo na Estratégia Saúde da Família (ESF), considerando-a uma reorientação do modelo assistencial fundado na atenção básica, de acordo com os princípios do Sistema Único de Saúde (SUS). O antigo modelo tradicional, centrado no padrão biomédico e na assistência à demanda sem ênfase às ações programáticas, vem sendo substituído pela ESF, cujas atribuições fundamentais constituem ações de proteção e promoção à saúde dos indivíduos e suas famílias. ${ }^{13}$

$O$ trabalho das equipes de ESF, geralmente formadas por um médico generalista, um enfermeiro, um auxiliar ou técnico de enfermagem e até 12 agentes comunitários de saúde (ACS), prioriza a assistência a alguns grupos populacionais biologicamente mais vulneráveis a agravos como, por exemplo, gestantes e crianças menores de dois anos; 11 destacando-se entre as ações desenvolvidas pelas equipes de saúde a assistência maternoinfantil, que envolve a promoção e o manejo do AM. 12

Ancorando-se nos benefícios do AM, na sua duração, ainda aquém das recomendações da Organização Mundial da Saúde (OMS) e a influência dos profissionais de saúde em sua prevalência, foi realizado o presente estudo com o objetivo de avaliar e comparar o conhecimento e a qualidade do manejo do AM entre profissionais de saúde atuando na ESF e em unidades básicas de saúde (UBS) com modelo assistencial tradicional, no Município de Rio Grande/RS. A principal hipótese deste estudo era de que os profissionais que atuavam na ESF tinham um conhecimento maior sobre o AM e ofereciam o manejo de forma mais adequada do que aqueles que atuavam em UBS do modelo assistencial tradicional.

\section{Métodos}

Trata-se de um estudo transversal, realizado em Rio Grande, município localizado no extremo sul do Rio Grande do Sul, distante $317 \mathrm{~km}$ da capital do Estado. Em 2.709,5 $\mathrm{km}^{2}$ de área, abriga 197.228 habitantes. ${ }^{13}$ Atualmente, tem como referência um polo naval e indústrias petroquímicas e de fertilizantes, marcantes em sua economia. Conta com dois hospitais gerais, sendo um deles universitário, o qual possui a Iniciativa Hospital Amigo da Criança, além de outra instituição especializada em cardiologia. Dispõe também de uma rede de 34 UBS, sendo 19 com ESF, totalizando 26 equipes de ESF. Apresentou cerca de 2500 nascidos vivos/ano e seu coeficiente de mortalidade infantil foi de 13,8 mortes a cada 1000 nascidos vivos no ano de 2012.14

Foram convidados a participar do estudo todos 
os médicos, enfermeiros, técnicos e auxiliares de enfermagem e ACS da rede básica de saúde da zona urbana de Rio Grande, sendo excluídos os profissionais em licença-maternidade ou licençasaúde. Em função disso, não foi necessário realizar um cálculo de tamanho de amostra.

A coleta de dados ocorreu nas 24 UBS da zona urbana do município, 13 com ESF e 11 sem ESF, de junho a outubro de 2012, tendo sido excluídas as 10 UBS da zona rural. As entrevistas foram conduzidas por uma enfermeira e seis acadêmicas do curso de Enfermagem da Universidade Federal do Rio Grande, previamente treinadas segundo o manual de instruções elaborado especificamente para esta pesquisa. Visitaram-se todas as UBS a fim de explicar aos responsáveis os objetivos da pesquisa e agendar as entrevistas com aplicação de um questionário, com questões abertas e fechadas, dividido em três blocos descritos a seguir.

O bloco A contemplou as informações individuais dos participantes como o sexo, idade, possuir filhos, e em caso positivo se foram amamentados, o modelo de atenção no serviço onde trabalhavam, a realização de pós-graduação com enfoque em saúde pública - para profissionais de nível superior, o tempo de formação, tempo de atuação na atenção básica e na ESF, realização de capacitação em AM, além de acesso ao Caderno de Atenção Básica n ${ }^{\circ} 23$ do MS.10

Os desfechos de conhecimento e manejo do AM foram medidos considerando-se questões abertas e fechadas dos Blocos B e C, que permitiram a construção de escores baseados nas recomendações do Caderno de Atenção Básica no 23 do MS.10

O Bloco B englobou o conhecimento dos profissionais em relação a vantagens do AM, tanto para a mãe quanto para a criança, bem como duração da amamentação, importância do AM, produção do leite materno, uso de chupeta e mamadeira durante a amamentação, alimentação da nutriz, ordenha, armazenamento e aquecimento do leite materno, maneiras de oferecer o leite ordenhado aos lactentes e contraindicações do AM.

Por fim, o Bloco C abrangeu questões sobre o domínio de técnicas de amamentação, pontos-chave sobre posicionamento e pega, prevenção e manejo dos problemas centrais relacionados à amamentação e ao manejo do AM em situações especiais. Considerou-se como escore de conhecimento em AM a pontuação decorrente das assertivas dos blocos B e C, e o escore de manejo foi medido levando-se em conta as questões do bloco C. As perguntas abertas foram validadas corretas de acordo com as recomendações do MS. 10

Nas questões objetivas, a codificação das respostas foi feita da seguinte forma: 2 (correta) ou zero (incorreta); já nas perguntas abertas, a codificação foi: 2 (correta), 1 (parcialmente correta) ou zero (incorreta). Para isso, foram utilizados como ponto de corte os mesmos valores empregados no estudo de Silvestre et al.,15 devido à metodologia semelhante. Quanto ao conhecimento e manejo julgaram-se satisfatórios os escores acima de $80 \%$; regulares, aqueles entre $79,9 \%$ e $50 \%$; e insatisfatórios os escores abaixo de $50 \%$.

Elaboraram-se três questionários diferentes, de acordo com o nível de formação de cada categoria profissional: um para os de nível superior (enfermeiros e médicos), outro para auxiliares e técnicos de enfermagem, e o último para os ACS. Os questionários foram previamente testados quanto à sua adequação e seu preenchimento em duas UBS da cidade de Pelotas, situada a $60 \mathrm{~km}$ de Rio Grande, sendo uma UBS tradicional e outra com ESF.

Utilizou-se o Programa Epi Info, versão 6.04, para dupla digitação dos questionários. Após, os dados foram transferidos ao pacote estatístico Stata, versão 11.0 , com vistas a efetuar as análises. As perguntas abertas foram transformadas em variáveis categóricas. A comparação das proporções foi avaliada mediante aplicação do Teste Exato de Fisher. Na interpretação dos resultados dos escores de conhecimento e manejo, empregou-se o Teste t de Student visando à comparação das médias. Finalmente, procedeu-se a uma análise de mediação, 16 com o intuito de verificar se uma variável altera o efeito de outra. A análise foi feita controlando o efeito do modelo de atenção para a realização de capacitação em AM e a leitura do manual do MS. Este tipo de análise foi feito para testar o efeito do modelo de atenção sobre os escores de conhecimento e de manejo que não se atribui à realização de capacitação em AM nem à leitura do manual.

Além disso, testou-se se havia interação entre as variáveis analisadas e o modelo de atenção, não sendo constatada modificação significativa $(p<0,10)$ nos resultados. Em vista disso, as análises não foram estratificadas pelo modelo de atenção e o nível de significância adotado foi de $5 \%$ para testes bicaudais.

Este trabalho obteve aprovação pelo Comitê de Ética em Pesquisa na Área da Saúde da Universidade Federal do Rio Grande, conforme o Parecer $n^{\circ}$ $184 / 2011$. 


\section{Resultados}

Do total de 296 profissionais, 269 (90,9\%) aceitaram participar do estudo. Houve a recusa de seis $(2,0 \%)$ médicos, seis $(2,0 \%)$ auxiliares e técnicos de enfermagem, além de nove $(3,0 \%)$ ACS. Foram consideradas perdas e excluídos cinco $(1,7 \%)$ ACS e um auxiliar de enfermagem $(0,3 \%)$, pois estavam em licença-saúde ou maternidade, configurando-se, assim, a população: 37 médicos, 46 enfermeiros, 74 auxiliares ou técnicos de enfermagem e 112 ACS (Tabela 1).

Houve predomínio do sexo feminino em todas as categorias profissionais, com 59,5\%, 93,5\%, 82,4\% e $94,6 \%$, respectivamente. A maior média de idade ocorreu entre os médicos: 48,5 anos $(\mathrm{DP}=8,8)$; e a menor entre os ACS: 38,5 anos ( $D P=9,6)$. A idade maior que 20 anos de formação predominou entre os médicos (70,3\%), com 48,6\% atuando entre 10 e 20 anos na atenção básica e $56,2 \%$ dos que trabalhavam na ESF atuavam havia mais de cinco anos nessa estratégia. A maioria $(52,2 \%)$ dos enfermeiros tinha entre 10 e 20 anos de formação, $45,7 \%$ com menos de dez anos de atuação na atenção básica e, entre os que trabalhavam na ESF, $60 \%$ tinham atuação entre cinco e dez anos. Pouco mais de $39 \%$ dos auxiliares e técnicos de enfermagem havia concluído o curso entre 10 e 20 anos, com $47,5 \%$ atuando havia menos de dez anos na atenção básica, e, entre os que trabalhavam na ESF, $50 \%$ atuavam entre cinco e dez anos. Para os ACS, a maioria $(87,4 \%)$ tinha concluído o curso de ACS menos de dez anos atrás; $84,8 \%$ possuíam menos de dez anos na atenção básica; e, entre os que trabalhavam na ESF, $70,7 \%$ atuavam havia menos de cinco anos (Tabela 2).

A Tabela 3 mostra o perfil de desempenho dos profissionais no conhecimento e no manejo do AM, conforme os modelos de atenção tradicional e da ESF. De maneira geral, os profissionais da ESF obtiveram melhores desempenhos tanto no escore de conhecimento quanto no escore de manejo, quando comparados aos profissionais do modelo tradicional. Foi observada uma diferença estatisticamente signi- ficativa entre os escores de conhecimento de acordo com o modelo de atenção para os enfermeiros e os auxiliares e técnicos de enfermagem, ao passo que no escore de manejo essa diferença foi observada para os mesmos profissionais, além dos ACS. Dos 269 profissionais, somente $22(8,2 \%)$ e $50(18,6 \%)$ apresentaram desempenho satisfatório nos escores de conhecimento e manejo, respectivamente. Ao dividirmos os profissionais de acordo com o modelo de atenção, percebemos que na ESF somente 17 $(11,2 \%)$ e $36(23,7 \%)$ apresentaram desempenho adequado nos escores de conhecimento e manejo, respectivamente, enquanto que os profissionais do modelo tradicional apresentaram $5(4,3 \%)$ e 14 (12\%), nessa ordem.

$\mathrm{Na}$ Tabela 4, verifica-se que as diferenças entre as médias do escore de conhecimento em AM para os médicos foram significativamente associadas ao modelo de atenção, ao sexo, à realização de pósgraduação e à leitura do manual do MS. Para os enfermeiros, auxiliares e técnicos de enfermagem, observa-se associação significante quanto ao modelo de atenção, à realização de capacitação em $\mathrm{AM}$ e à leitura do Manual do MS e para os ACS, quanto ao modelo de atenção, ao sexo, à realização de capacitação em AM e à leitura do Manual do MS.

$\mathrm{Na}$ Tabela 5, verifica-se que as diferenças entre as médias do escore de manejo em AM para os médicos foram significativamente associadas ao modelo de atenção e à leitura do Manual do MS. Em relação aos enfermeiros somente não apresentaram associação significativa as variáveis ter filhos e a amamentação destes. Para os auxiliares e técnicos de enfermagem, associaram-se significativamente a realização de capacitação em AM e a leitura do Manual do MS, enquanto que para os ACS o modelo de atenção e a realização de capacitação em AM associaram-se significativamente com médias mais elevadas no escore de manejo.

Para ambos os escores dos enfermeiros e dos auxiliares e técnicos de enfermagem, o efeito do modelo de atenção foi mediado pela realização de capacitação em AM. 


\section{Tabela 1}

População de estudo de acordo com cada categoria profissional atuante na rede básica de saúde da cidade do Rio Grande/RS, 2012 ( $N=296)$.

\begin{tabular}{|c|c|c|c|c|c|c|c|}
\hline \multirow{2}{*}{ Categorias profissionais } & \multirow{2}{*}{$\begin{array}{c}\begin{array}{c}\text { População } \\
\text { elegível }\end{array} \\
n\end{array}$} & \multicolumn{2}{|c|}{ Recusas } & \multicolumn{2}{|c|}{ Perdas } & \multicolumn{2}{|c|}{$\begin{array}{l}\text { População } \\
\text { estudada }\end{array}$} \\
\hline & & $\mathrm{n}$ & $\%$ & $\mathrm{n}$ & $\%$ & $\mathrm{n}$ & $\%$ \\
\hline Médicos & 43 & 6 & 14,0 & 0 & - & 37 & 86,0 \\
\hline Enfermeiros & 46 & 0 & - & 0 & - & 46 & 100,0 \\
\hline Auxiliares e técnicos de enfermagem & 81 & 6 & 7,4 & 1 & 1,2 & 74 & 91,4 \\
\hline Agentes comunitários de saúde & 126 & 9 & 7,1 & 5 & 4,0 & 112 & 88,9 \\
\hline Total & 296 & 21 & 7,4 & 6 & 2,0 & 269 & 90,9 \\
\hline
\end{tabular}

Tabela 2

Características da população estudada de acordo com cada categoria profissional atuante na rede básica de saúde da cidade do Rio Grande/RS, 2012 ( $\mathrm{N}=269$ ).

\begin{tabular}{|c|c|c|c|c|c|c|c|c|}
\hline \multirow[t]{2}{*}{ Variáveis } & \multicolumn{2}{|c|}{ Médicos } & \multicolumn{2}{|c|}{ Enfermeiros } & \multicolumn{2}{|c|}{$\begin{array}{l}\text { Auxiliares e } \\
\text { técnicos de } \\
\text { enfermagem }\end{array}$} & \multicolumn{2}{|c|}{$\begin{array}{l}\text { Agentes comunitários } \\
\text { de saúde }\end{array}$} \\
\hline & $\mathrm{n}$ & $\%$ & $\mathrm{n}$ & $\%$ & $\mathrm{n}$ & $\%$ & $\mathrm{n}$ & $\%$ \\
\hline \multicolumn{9}{|l|}{ Modelo de atenção } \\
\hline UBSF & 13 & 35,1 & 18 & 39,1 & 23 & 31,1 & 98 & 87,5 \\
\hline UBS Tradicional & 24 & 64,9 & 28 & 60,9 & 51 & 68,9 & 14 & 12,5 \\
\hline \multicolumn{9}{|l|}{ Sexo } \\
\hline Masculino & 15 & 40,5 & 3 & 6,5 & 13 & 17,6 & 6 & 5,4 \\
\hline Feminino & 22 & 59,5 & 43 & 93,5 & 61 & 82,4 & 106 & 94,6 \\
\hline \multicolumn{9}{|c|}{ Tempo de Formação (anos) } \\
\hline$<10$ & 4 & 10,8 & 10 & 21,7 & 20 & 27,0 & 98 & 87,5 \\
\hline Entre 10 e 20 & 7 & 18,9 & 24 & 52,2 & 39 & 39,2 & 14 & 12,5 \\
\hline$>20$ & 26 & 70,3 & 12 & 26.1 & 25 & 33,8 & 0 & - \\
\hline \multicolumn{9}{|c|}{$\begin{array}{l}\text { Tempo de trabalho na atenção básica } \\
\text { (anos) }\end{array}$} \\
\hline$<10$ & 11 & 29,7 & 21 & 45,7 & 35 & 47,3 & 95 & 84,8 \\
\hline Entre 10 e 20 & 18 & 48,6 & 22 & 47,8 & 34 & 45,9 & 17 & 15,2 \\
\hline Entre 20 e 30 & 4 & 10,8 & 0 & - & 5 & 6,8 & 0 & - \\
\hline$>30$ & 4 & 10,8 & 3 & 6,5 & 0 & - & 0 & - \\
\hline \multicolumn{9}{|c|}{ Tempo de trabalho na ESF (anos) } \\
\hline$<5$ & 7 & 43,8 & 6 & 30,0 & 9 & 34,6 & 70 & 70,7 \\
\hline Entre 5 e 10 & 6 & 37,5 & 12 & 60,0 & 13 & 50,0 & 16 & 16,2 \\
\hline$>10$ & 3 & 18,8 & 2 & 10,0 & 4 & 15,4 & 13 & 13,1 \\
\hline
\end{tabular}




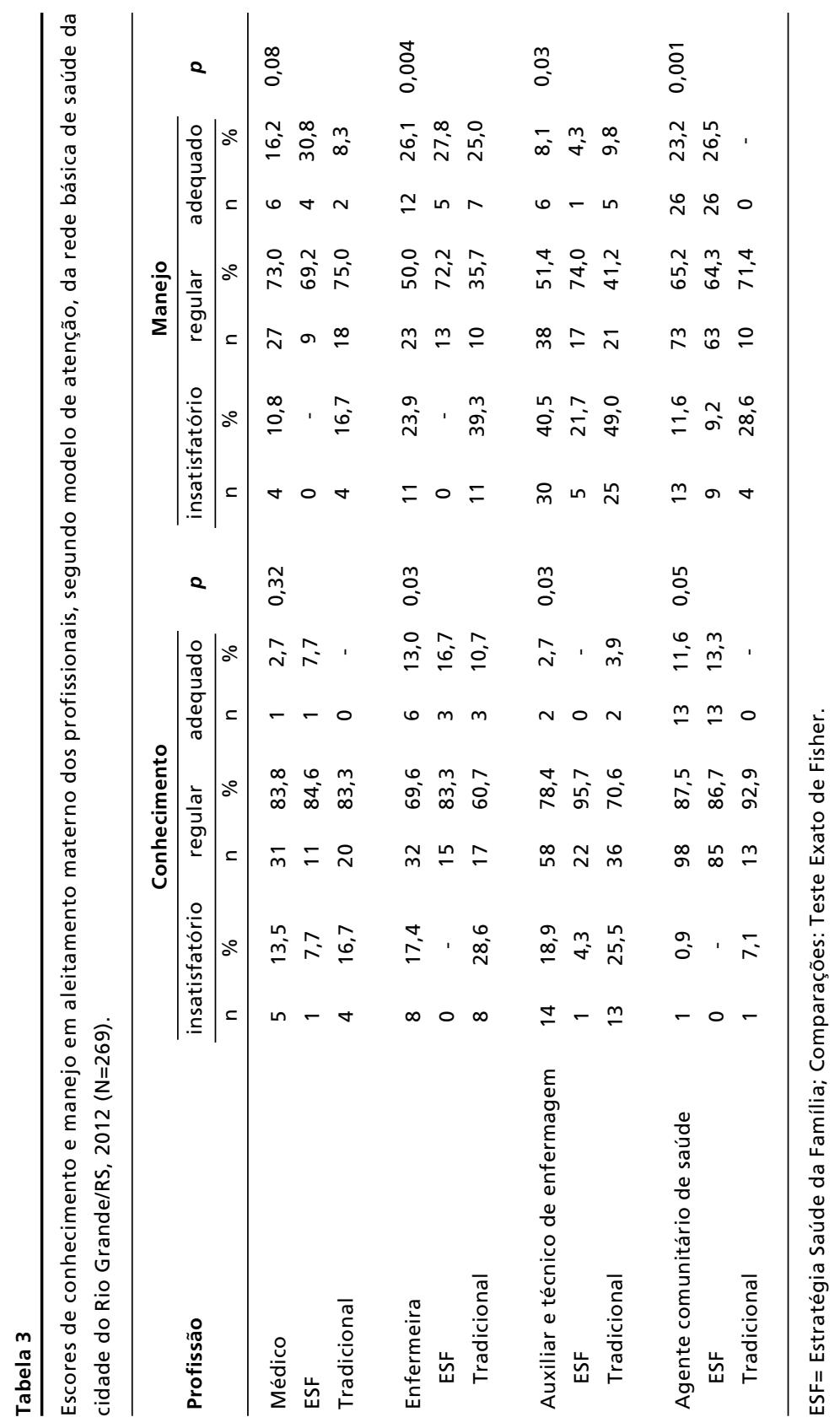




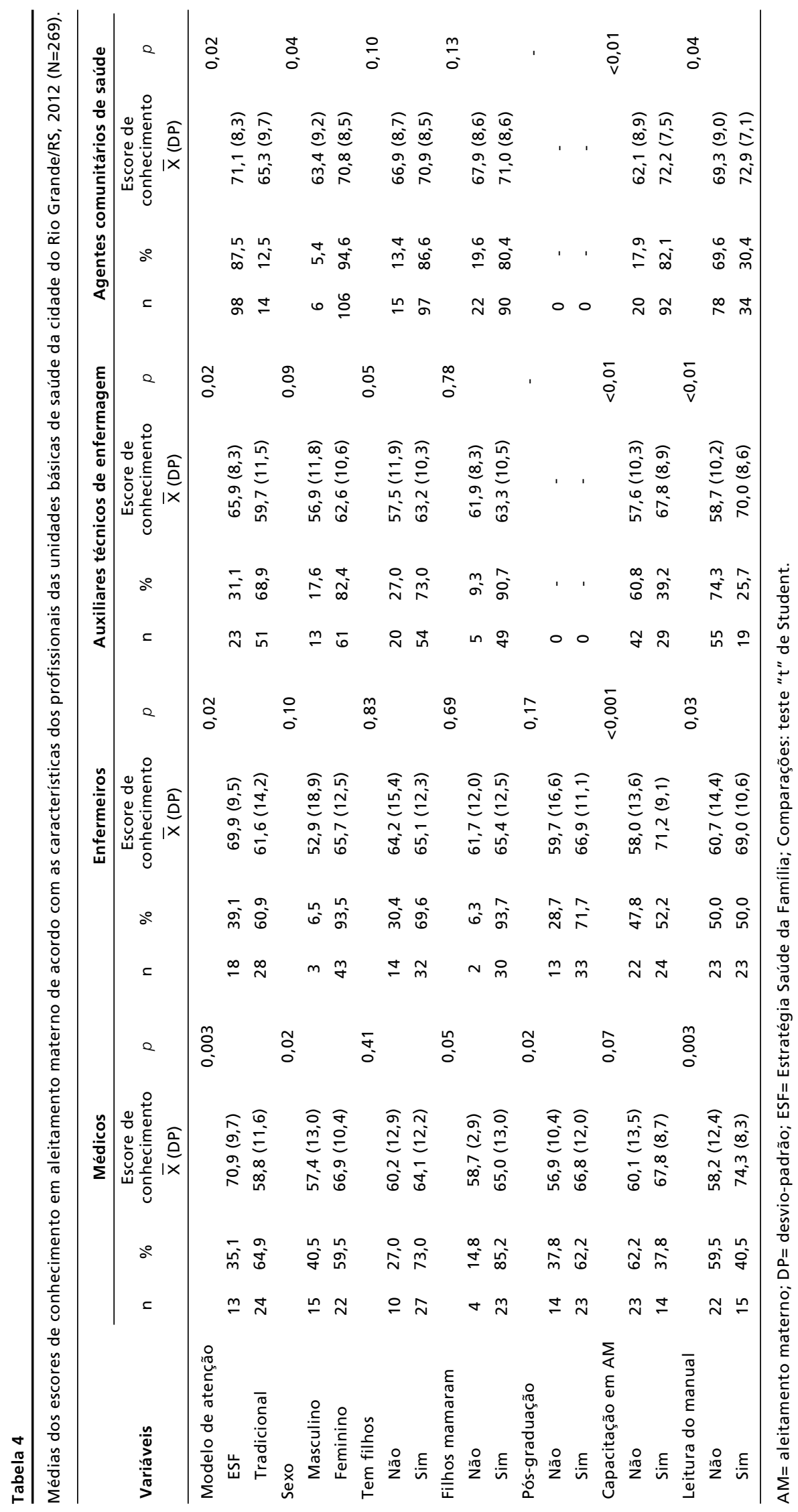




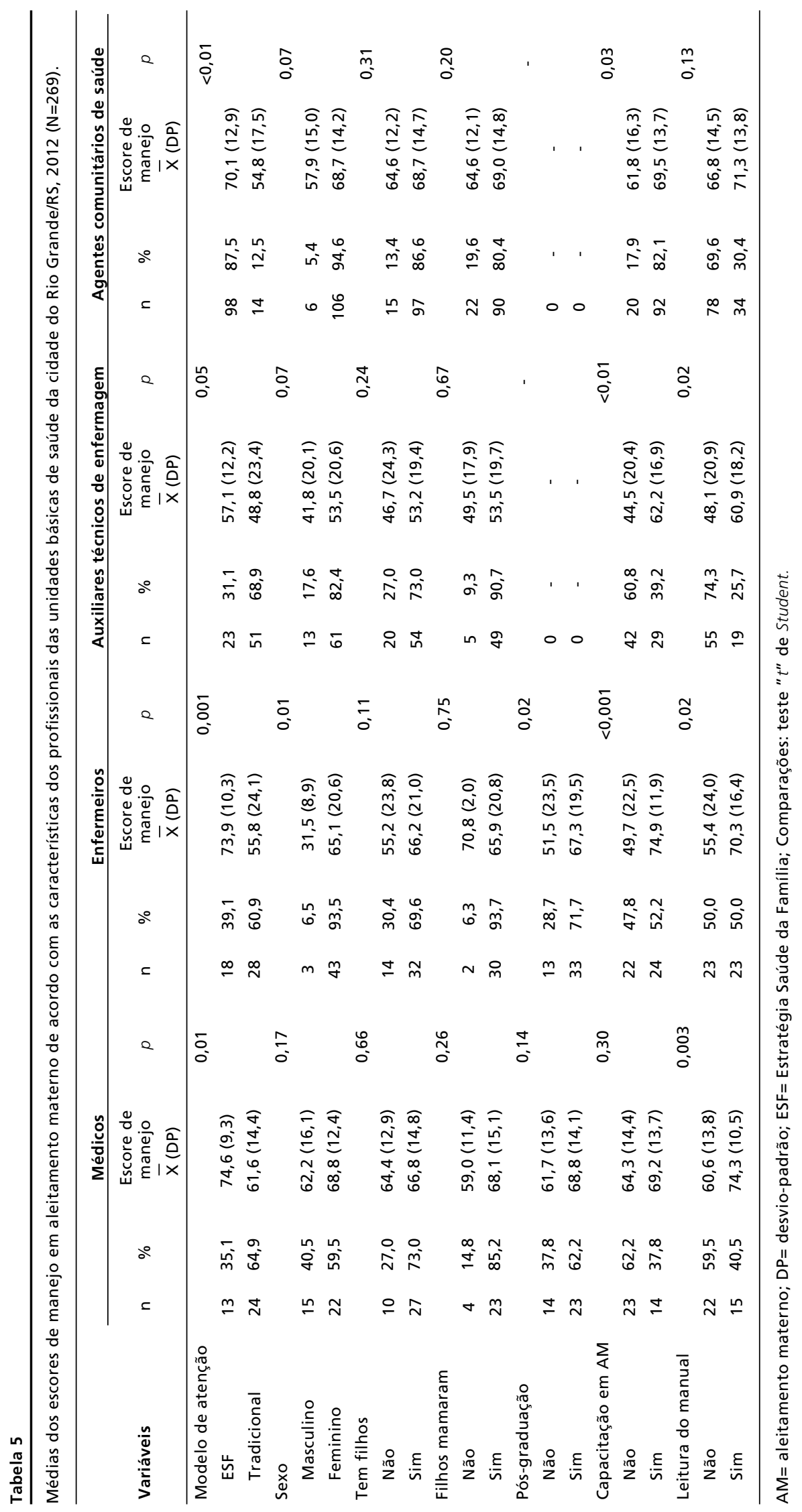




\section{Discussão}

Este estudo incluiu profissionais atuantes nas UBS de modelo tradicional e também nas com ESF, permitindo a obtenção de abrangente panorama sobre conhecimentos e práticas na área do $\mathrm{AM}$, no município em questão. Foi constatado que os profissionais da ESF alcançaram médias de acerto mais elevadas, tanto no conhecimento quanto no manejo do AM, quando comparados aos do modelo tradicional. Entretanto, essa superioridade não denota adequação durante a prática, pois somente 17 $(11,2 \%)$ e cinco $(4,3 \%)$ daqueles os quais exerciam suas atividades na ESF apresentaram desempenho satisfatório nos escores de conhecimento e manejo, respectivamente. A realização de capacitação em AM associou-se significativamente com médias mais elevadas nos escores de conhecimento e manejo do AM, destacando-se como um diferencial para todas as categorias, excetuando-se os médicos.

Não foram encontrados estudos que avaliassem o conhecimento e o manejo em AM dos profissionais de saúde explorando diferenças conforme as variáveis desta pesquisa. Embora a literatura disponha de alguns estudos sobre a temática, a maioria deles aborda as atividades exercidas pelos profissionais na promoção do $\mathrm{AM}$ ou aferição contínua do seu conhecimento após participação em atividades educativas. 12,17

A análise de desempenho nos testes de conhecimento e manejo do AM permite identificar que os profissionais em sua maioria obtiveram desempenho regular para ambos os escores. A baixa porcentagem de profissionais com desempenho adequado é preocupante. Considerando que esses profissionais atendem gestantes e puérperas, oferecendo orientações e esclarecimentos quanto à técnica correta da amamentação, bem como auxiliando na resolução de problemas os quais podem ocorrer durante a lactação, esta porcentagem está aquém do esperado. A escassez de conhecimento e habilidades de profissionais de saúde para apoiar o AM tem sido apontada como uma das principais causas de falha no início e na manutenção dessa prática. ${ }^{12,18,19}$

Entretanto, estudo realizado em Uberaba/MG, 20 mostrou que os profissionais de saúde frequentemente orientavam as gestantes acerca do AM, independentemente do nível de conhecimento, favorecendo a repercussão negativa na manutenção do AM, pois informações incorretas ou incompletas podem favorecer o desmame precoce. Parece haver consenso quanto à necessidade de capacitar os profissionais de saúde a fim de habilitá-los a prestarem apoio competente e seguro às mães que amamentam, sob pena das orientações corretas sobre o AM não serem cumpridas. 21,22

Todos os profissionais atuantes na ESF obtiveram médias superiores aos do modelo tradicional nos escores de conhecimento e manejo. Observou-se, porém, diferença estatística significativa entre ambos os escores e o modelo de atenção para todas as profissões, exceto para auxiliares e técnicos de enfermagem em relação ao escore de manejo do AM, cujo valor $p$ ficou no limiar da significância $(p=0,05)$.

Cruz et al.,11 em estudo transversal, ressaltaram serem todas as orientações sobre amamentação significativamente mais referidas por moradoras de áreas de ESF do que por aquelas de UBS tradicional. Com isso demonstraram ter o modelo de atenção forte associação com as informações concedidas pelos profissionais. Também é relevante salientar que o governo federal considera a ESF a alternativa ideal para transformar a realidade existente por meio de práticas de saúde solidárias, acolhedoras e, consequentemente, mais efetivas e resolutivas que as apresentadas pelo modelo assistencial tradicional ainda existente. 23

Os profissionais que realizaram a capacitação em AM obtiveram as maiores médias de acertos comparados com aqueles os quais não a realizaram, sendo essa diferença estatisticamente significativa a todos os profissionais, excetuando-se os médicos. Identificamos que a realização de capacitação em AM tem forte efeito sobre o modelo de atenção para enfermeiros, auxiliares e técnicos de enfermagem, enquanto que para médicos e ACS a capacitação não media o efeito do modelo de atenção sobre os desfechos analisados. Esse dado corrobora a importância da capacitação dos profissionais de saúde de modo a possibilitar o embasamento teórico das suas ações, tornando-as mais efetivas em prol do aleitamento.

Observou-se neste estudo que menos da metade dos médicos e dos auxiliares e técnicos de enfermagem tinham realizado alguma capacitação na temática, enquanto que mais de $80 \%$ dos ACS já haviam sido envolvidos em algum tipo de processo educativo. Marques et al.22 destacam ser necessária a implementação de políticas de capacitação e educação permanente em amamentação para o desenvolvimento de ações mais resolutivas a favor do aleitamento.

Pesquisa realizada em Botucatu/SP, 24 com 54 ACS atuantes na ESF, igualmente testemunhou melhor desempenho do conhecimento e da prática do AM após realização de ação educativa. Resultado similar foi encontrado em estudo feito em São Carlos/SP, 25 em 2005, com profissionais de nível 
superior os quais trabalhavam em UBS da zona urbana do município. As duas categorias que obtiveram um melhor desempenho no teste de conhecimentos foram aquelas as quais relataram ter cursado duas capacitações ou mais na área. Portanto, insiste-se que programas específicos de capacitação devem incluir todos os grupos de profissionais, de forma a tornar mais expressiva sua participação na melhoria das taxas de AM.

As mulheres entrevistadas atingiram médias mais altas que as dos homens em ambos os desfechos para todos os profissionais. Entretanto, a variável sexo associou-se significativamente com o escore de conhecimento somente para médicos e $\mathrm{ACS}$, enquanto que no escore de manejo apenas para enfermeiros. Ciconi et al. 12 pesquisaram acerca do assunto em Francisco Morato/SP, com integrantes da ESF, constatando ser a maioria dos profissionais do sexo feminino, embora a associação entre a variável sexo e o número de acertos não ter sido significativa. A associação com o sexo feminino pode ter ocorrido porque as mulheres receberam mais capacitação em AM e leram mais o manual do que os homens, além disso, deve-se considerar que há mais profissionais de saúde mulheres tanto no modelo tradicional quanto na ESF.

As profissionais terem filhos ou terem experiência prévia com a amamentação não indicou associação significativa com nenhum dos escores, quando se esperava que a vivência do processo de amamentação, experiência intrinsecamente feminina, conferisse um conhecimento mais amplo sobre $\mathrm{o}$ assunto. Parece, portanto, que apenas a vivência e a experiência prévia com o AM não denotam competência para manejá-lo, podendo contribuir para a perpetuação de crenças, mitos e falsas verdades. 20 Todavia, resultados de pesquisa revelaram que as profissionais seguem mais a sua própria experiência de mãe ou de observar outras mães do que se fundamentarem nos conhecimentos científicos. ${ }^{26} \mathrm{O}$ conhecimento técnico é uma ferramenta crucial na orientação do AM, bem como no manejo de situações de risco para o desmame precoce.

Quanto à pós-graduação com enfoque na saúde pública, esperavam-se escores mais altos dos profissionais que a tivessem cursado se comparados com aqueles os quais não a cursaram. Tal diferença se confirmou, entretanto, associado notadamente a uma média mais elevada no escore de conhecimento para os médicos e de manejo para os enfermeiros. Segundo Parizoto et al.,27 entre 1999 e 2010, foi expressivo o número de dissertações e teses que tinham a amamentação ou o aleitamento como tema central da pesquisa na área da saúde, e, entre elas,
$20 \%$ eram proveniente da área da enfermagem. Apesar deste interesse sobre o tema, neste estudo, cursar a pós-graduação, isoladamente, não foi suficiente para demonstrar diferença quanto ao manejo para os médicos e conhecimento para os enfermeiros.

O Manual do MS sobre nutrição infantil, que aborda o AM10 é uma excelente ferramenta voltada ao conhecimento amplo acerca da sua teoria e prática. A leitura desse material revelou diferenças entre as médias de ambos os desfechos a de todos os profissionais, à exceção dos ACS no escore de manejo do AM.

Vale ressaltar a existência de algumas limitações no presente trabalho. Por se constituir um estudo transversal, os resultados aqui apresentados refletem estritamente o período no qual os dados foram coletados. Sendo importante, ainda, ressaltar a possibilidade de que algumas análises não apresentaram significância estatística devido ao tamanho da amostra. Além disso, médicos com menos conhecimento sobre AM podem ter sido justamente aqueles que se recusaram a participar da pesquisa, contribuindo assim com o melhor desempenho dessa categoria profissional.

Os resultados deste estudo sinalizam a possibilidade de melhorar o desempenho dos profissionais da atenção primária, tanto do modelo de atenção tradicional quanto da ESF, por meio da realização de capacitações com a temática em foco. Além disso, é necessário que busquem embasamento teórico com o propósito de orientar, apoiar e promover o AM. Um instrumento bastante completo com tal finalidade é o Manual do MS, o qual aborda o AM,10 tema do presente estudo. Outra estratégia viável seria a adesão à Iniciativa Unidade Básica Amiga da Amamentação, pois ela delineia um importante papel de suporte que essas unidades, em conjunto com os hospitais, podem desempenhar a fim de tornar o AM uma prática universal e contribuir notavelmente para a saúde e bem-estar dos bebês, suas mães, família e comunidade local. 28

Para os enfermeiros, auxiliares e técnicos de enfermagem, o resultado dos escores de conhecimento e manejo do AM dependeu da realização de capacitação em AM, ou seja, esta exerce um forte efeito sobre o modelo de atenção, enquanto que para médicos e ACS ela não atuou como mediadora. Portanto, ao se analisarem os dados evidenciam-se que as lacunas relacionadas tanto ao conhecimento quanto ao manejo não são específicas, ou seja, os profissionais desconheciam inúmeros pontos relacionados à temática.

Desse modo, possíveis intervenções para a 
capacitação e sensibilização dos profissionais referentes à temática em foco deverão ser efetivadas, visando formar equipes qualificadas e compro-

\section{Referências}

1. World Health Organization (WHO), Regional Office for the Americas. Indicators for Assessing Infant and Young Child Feeding Practices, Washington; 2008. [acesso em 04 mai 2015]. Disponível em: http://whqlibdoc. who.int/publications/2008/9789241596664_eng.pdf.)

2. Victora CG, huttly SR, Fuchs SC, Nobre LC, Barros FC. Deaths due to dysentery, acute and persistent diarrhoea among Brazilian infants. Acta Pediatr. 1992; 381: 7-11.

3. Brasil. Ministério da Saúde. Centro Brasileiro de Análise e Planejamento. Pesquisa Nacional de Demografia e Saúde da Criança e da Mulher - PNDS 2006: dimensões do processo reprodutivo e da saúde da criança. Capítulo 10. (Série G. Estatística e Informação em Saúde). Brasília, DF; 2009

4. Brasil. Ministério da Saúde. Secretaria de Atenção à saúde. Departamento de Ações Programáticas e Estratégicas. II Pesquisa de Prevalência de Aleitamento Materno nas Capitais Brasileiras e Distrito Federal. Brasília, DF; 2009.

5. Vieira GO, Martins CC, Vieira TO, Oliveira NF, Silva LR. Fatores preditivos da interrupção do aleitamento materno exclusivo no primeiro mês de lactação. J Pediatr. 2010; 86 (5): 441-4

6. Vianna RP, Rea MF, Venancio SI, Escuder MM. A prática de amamentar entre mulheres que exercem trabalho remunerado na Paraíba, Brasil: um estudo transversal. Cad Saúde Pública. 2007; 23 (10): 2403-09.

7. Machado MCM, Assis KF, Oliveira FCC, Ribeiro AQ, Araújo RMA, Cury AF, Priore SE, Franceschini SCC. Determinantes do abandono do aleitamento materno exclusivo: fatores psicossociais. Rev Saúde Pública. 2014; 48 (6): 985-94.

8. Haga SM, Ulleberg P, Slinning K, Kraft P, Steen TB, Staff A. A longitudinal study of postpartum depressive symptoms: multilevel growth curve analyses of emotion regulation strategies, breastfeeding self-efficacy, and social support. Arch Womens Ment Health. 2012; 15(3): 175-84.

9. Monteiro JCS, Nakano AMS, Gomes FA. O aleitamento materno enquanto uma prática construída: Reflexões acerca da evolução histórica da amamentação e desmame precoce no Brasil. Invest Educ Enferm. 2011; 29 (2): 315-22.

10. Brasil. Ministério da Saúde. Secretaria de Atenção à Saúde. Departamento de Atenção Básica. Saúde da criança: nutrição infantil, aleitamento materno e nutrição complementar. (Cad Atenção Básica, nº 23). Brasília, DF; 2009.

11. Cruz SH, Germano JA, Tomasi E, Facchini LA, Piccini RX, Thumé E. Orientações sobre amamentação: vantagem do Programa de Saúde da Família em municípios gaúchos com mais de 100.000 habitantes no âmbito do PROESF. Rev Bras Epidemiol. 2010; 13 (2): 259-67.

12. Ciconi RCV, Venancio SI, Escuder MML. Avaliação dos conhecimentos de equipes do Programa de Saúde da Família sobre o manejo do aleitamento materno em um metidas com a promoção, proteção e apoio da amamentação, para a melhoria nos índices do AM. município da região metropolitana de São Paulo. Rev Bras Saúde Matern Infant. 2004; 4 (2): 193-202.

13. IBGE (Instituto Brasileiro de Geografia e Estatística). IBGE Cidades. 2010. [acesso em 10 fev 2015]. Disponível em: http://www.ibge.gov.br/cidadesat/link.php?uf=rs

14. SMS (Secretaria Municipal de Saúde). Número de nascidos vivos e coeficiente de mortalidade infantil do Município do Rio Grande/RS em 2012. (Informação obtida com a Coordenação da Atenção Básica do Município do Rio Grande/RS em maio de 2013).

15. Silvestre PK, Carvalhaes MABL, Venâncio SI, Tonete VLP, Parada CMGL. Conhecimentos e práticas de profissionais de saúde sobre aleitamento materno em serviços públicos de saúde. Rev Latino-Am Enferm. 2009; 17 (6): 953-60.

16. Mackinnon DP, Fairchild AJ, Fritz MS: Mediation analysis. Ann Rev Psychol. 2007; 58: 593-614.

17. Caldeira AP, Fagundes GC, Aguiar GN. Intervenção educacional em equipes do Programa de Saúde da Família para promoção da amamentação. Rev Saúde Pública. 2008; 42 (6): 1027-33.

18. Cummings M. Best practice standards for breastfeeding education: A baby friendly approach. Nurse Educ Today. 2008; 28 (8): 895-8.

19. Fujimori E, Nakamura E, Gomes MM, Jesus LA, Rezende Ma. Aspectos relacionados ao estabelecimento e à manutenção do aleitamento materno exclusivo na perspectiva de mulheres atendidas em uma unidade básica de saúde. Interface. 2010; 14 (33): 315-27.

20. Fonseca-Machado MO, Haas VJ, Stefanello J, Nakano MAS, Gomes-Sponholz F. Aleitamento materno: conhecimento e prática. Rev Esc Enferm USP. 2012; 46 (4): 80915.

21. Lundberg PC, Ngoc Thu TT. Breast-feeding attitudes and practices among Vietnamese mothers in Ho Chi Minh City. Midwifery. 2012; 28 (2): 252-7.

22. Marques RM, MENDES A. Atenção Básica e Programa de Saúde da Família (PSF): novos rumos para a política de saúde e seu financiamento? Ciênc. Saúde Coletiva. 2003; 8 (2): 403-15.

23. Costa GD, Cotta RMM, Ferreira MLSM, Reis JR, Franceschini SCC. Saúde da família: desafios no processo de reorientação do modelo assistencial. Rev Bras Enferm. 2009; 62 (1): 113-8.

24. Machado MCHS, Oliveira JS, Parada CMGL, Venâncio SI, Tonete VLP, Carvalhaes ABL. Avaliação de intervenção educativa sobre aleitamento materno dirigida a agentes comunitários de saúde. Rev. Bras. Saude Mater. Infant. 2010; 10 (4): 459-68.

25. Arantes CIS, Montrone AVG, Milioni DB. Concepções e conhecimento sobre amamentação de profissionais da atenção básica à saúde. Rev Eletr Enf [online]. 2008; 10 (4): 933-44. 
26. Marques ES, Cotta RMM, Franceschini SCC, Botelho MIV, Araújo RMA Junqueira TS. Práticas e percepções acerca do aleitamento materno: consensos e dissensos no cotidiano de cuidado numa Unidade de Saúde da Família. Physis. 2009; 19 (2): 439-55

27. Parizoto GM, Erdmann AL, Santos EKA, Andrade SR. Produção de teses e dissertação, sobre aleitamento materno, nos programas de pós-graduação em enfermagem. Acta Paul Enferm. 2012; 25 (4): 632-8

Recebido em 3 de novembro de 2014

Versão final apresentada em 11 de março de 2015

Aprovado em 1 de abril de 2015
28. Martins RMC, Montrone AVG. Implementação da Iniciativa Unidade Básica Amiga da Amamentação: educação continuada e prática profissional. Rev Eletr Enf. J Pediatr. 2009; 11 (3): $545-53$ 